\title{
Using Degradation-with-Jump Measures to Estimate Life Characteristics of Lithium-Ion Battery
}

\author{
Yin Shu ${ }^{1}$, Qianmei Feng ${ }^{2 *}$, Hao Liu $^{3}$ \\ ${ }^{1}$ Science and Technology Division, Corning Incorporated, Corning, NY 14831, USA \\ ${ }^{2}$ Department of Industrial Engineering, University of Houston, E206 Engineering Bldg.2, Houston, TX 77204, USA
}

${ }^{3}$ Department of Biostatistics, Indiana University, Indianapolis, IN 46202, USA

\begin{abstract}
Degradation-with-jump measures are time series data sets containing the information of both continuous and randomly jumping degradation evolution of a system. Traditional maximum likelihood estimation and Bayesian estimation are not convenient for such general jump processes without closed-form distributions. Based on general degradation models derived using Lévy driven non-Gaussian Ornstein-Uhlenbeck (OU) processes, we propose a systematic statistical method using linear programing estimators and empirical characteristic functions. The point estimates of reliability function and lifetime moments are obtained by deriving their explicit expressions. We also construct bootstrap procedures for the confidence intervals. Simulation studies for a stable process and a stable driven OU process are performed. In the case study, we use a general Lévy process to fit the Li-ion battery life data, and then estimate the reliability and lifetime moments of the battery. By integrally analyzing degradation data series embedded with jump measures, our work provides the efficient and precise estimation for life characteristics.
\end{abstract}

Keywords: Non-Gaussian Ornstein-Uhlenbeck, Autoregression (AR) models, Jump measures, Linear programing estimators, Empirical characteristic functions, Bootstrap, Li-ion battery

*qfeng@central.uh.edu

This is the author's manuscript of the article published in final edited form as:

Shu, Y., Feng, Q., \& Liu, H. (2019). Using Degradation-with-Jump Measures to Estimate Life Characteristics of Lithium-Ion Battery. Reliability Engineering \& System Safety, 106515. https://doi.org/10.1016/j.ress.2019.106515 


\section{Introduction}

Unavoidable degradation is one of the major failure mechanisms of many systems. Such degradation in critical engineering systems (e.g., wind turbines, drilling equipments, power/smart grids, and mechanical devices) takes the form of aging, corrosion, erosion, fatigue crack, deterioration or wear that may lead to the loss of structural integrity and catastrophic failure when it hits a boundary. With advanced measurement tools such as sensors, degradation data can be measured and collected effectively and economically, e.g., the Li-ion battery capacity data $[8,23,44]$, the integrated circuit propagation delay data [9], the metal fatigue-crack-growth data [26], and the transistor gain data [42]. The degradation data series over the life cycle reflect the evolution of the system's health state that contain more information than the sparse failure time data for reliable systems. Recently, the reliability estimation/prediction based on degradation measures [35-38] has gained popularity and become an effective approach, especially when it is costly and time-consuming to test and collect the failure time data for highly-reliable systems with advanced and evolving technologies.

In practice, a continuous degradation process commonly experiences complex jumps due to random damages caused by internal changes (physical, mechanical, thermal, electrical, or chemical) and external influences (temperature, pressure, humidity, or vibration). For example, the capacity loss and resistance increase of Li-ion batteries due to aging exhibit random jumps caused by thermal, electrical, and/or chemical changes. The complex jumps can be consecutive or sporadic, small or large, or their mixture. Observed from such degradation phenomena, degradation-with-jump measures are time series data sets containing the information of both continuous and randomly jumping degradation evolution of a system. In this paper, we develop a 
systematic approach to estimate the parameters in the degradation process using degradation-withjump measures, which can then be used to estimate reliability characteristics.

To analyze the degradation data, degradation models using appropriate stochastic processes need to be developed that can capture the temporal variability property in degradation. A Wiener process has been used for modeling the degradation without jumps that changes non-monotonically according to Gaussian laws [24, 43]. Some other degradation models without considering jumps were studied in $[13,41]$. A compound Poisson process has been applied to model a finite number of jumps that occur according to Poisson laws [18, 32]. A gamma process or an inverse Gaussian process has been widely used for modeling degradation processes that progress in one direction with an infinite number of jumps in any finite time interval, assuming the increments follow a gamma or an inverse Gaussian distribution [39, 40, 45-47]. These existing models, however, are limited in their assumptions on the certain-distributed and independent increments of the degradation process of interests.

To relax the assumption on certain-distributed increments, Lévy processes are appropriate to be used to construct degradation models. They form a broad class of jump processes represented by a general jump measure called Lévy measure $[2,29]$. This measure can model many different jump mechanisms being of either finite activity (a finite number of jumps in any time interval) or infinite activity (an infinite number of small jumps in any time interval) [7]. [14] is the first study to use Lévy processes in degradation analysis. Evolving by independent and stationary increments, Lévy processes have linear mean paths, i.e., the mean of a Lévy process is linear with respect to time. Thus they are suitable to model a class of stochastically continuous linear degradation paths with sporadic jumps that occur at random times and have random sizes [30].

To relax the assumption on independent increments, a functional extension of Lévy processes, 
non-Gaussian Ornstein-Uhlenbeck (OU) processes, can be used in degradation modeling. NonGaussian OU processes (OU processes driven by Lévy processes) are generalisation of ordinary OU processes by replacing the background driving Wiener processes with non-Gaussian Lévy processes (i.e., Lévy processes without Gaussian part; e.g., positive tempered stable processes) [35]. With the autoregressive structure, non-Gaussian OU processes evolve by dependent increments, resulting in nonlinear mean paths, which enables them to model a class of nonlinear degradation with random jumps [31].

To utilize the degradation models constructed using Lévy or non-Gaussian OU processes in [31], it is important to estimate the parameters of the underlying stochastic process using degradation data series, in order to further estimate/predict the reliability characteristics. The certain distribution of independent increments in existing models (Gaussian, Poisson, gamma, or inverse Gaussian) makes the statistical inference straightforward by using the likelihood function or Bayesian approach. For general Lévy and non-Gaussian OU processes, however, the traditional maximum likelihood estimation and Bayesian estimation are not convenient as the closed-form distributions are not available for such general jump processes. [15] provided a highly comprehensive and thorough treatment of Lévy processes in finance, covering Lévy models, simulation and estimation. [3] showed that it is not straightforward to implement traditional likelihood-based estimation procedures for the non-Gaussian OU-based model, although various moment-based methods are simple to use. [33] developed an efficient and explicit estimation procedure for non-Gaussian OU processes based on their characteristic functions, assuming that the marginal law belongs to a parametric family indexed by a parameter vector. The approach can deal with a general class of processes having both positive and negative jumps. The peculiar form of the characteristic functions of non-Gaussian $\mathrm{OU}$ processes and its relation with the 
characteristic functions of the underlying Lévy process were exploited in [34]. Based on the inversion of the characteristic function, they provided fast and reliable simulation procedures for OU processes. Simulation-based estimation procedures for non-Gaussian OU processes were discussed in $[21,22,28]$. The approximate results were often implemented since it is difficult to accurately simulate the jumps in the corresponding Lévy processes.

In this paper, we propose a systematic statistical inference method using linear programming estimators and empirical characteristic functions to estimate the parameters in the stochastic degradation models. The point estimates of reliability function and lifetime moments are then obtained by deriving their explicit expressions. Bootstrap procedures are also constructed for estimating the confidence intervals. To demonstrate the effectiveness of our proposed inference procedures, we use the capacity loss data of Li-ion batteries from randomized battery usage tests [8]. Our results are expected to provide accurate reliability estimation by constructing general stochastic models and providing systematic inference procedures.

The organization of this paper is as follows. In Section 2, we introduce our degradation and reliability models based on Lévy and non-Gaussian OU processes, respectively. In Section 3, we construct estimation procedures for the corresponding processes, including both point estimates and confidence intervals. Simulation studies are performed in Section 4 to demonstrate the advantages of the proposed method. Case study for battery degradation data is illustrated in Section 5. Finally, concluding remarks are made in Section 6.

\section{Stochastic Models}

We consider a system subject to degradation with random jumps, which is a process of stochastically continuous degradation with jumps that occur at random times and have random 
sizes. The scenarios of jumps include 1) consecutive small, 2) sporadic big/small, and 3) their mixture. We handle this complexity using a general random jump measure, instead of a certain probability measure that is used in the literature.

Lévy subordinators are the class of Lévy processes with nondecreasing sample paths. Based on Lévy-Itô decomposition [2], a Lévy subordinator $X_{s}(t)$ can be expressed as

$$
X_{s}(t)=b t+\int_{0<y<1} y(J(t, d y)-\nu(d y) t)+\int_{y \geq 1} y J(t, d y)
$$

where $b$ is a constant on $R^{+}$, and $J(t, d y)$ is a Poisson random/measure with an intensity measure (Lévy measure) $\nu(d y) t$, satisfying $\nu(\{0\})=0, \int_{R^{+}} \min \{1, y\} \nu(d y)<\infty$. In (1), the continuous degradation is modeled by $\left(b-\int_{0<y<1} y \nu(d y)\right) t \geq 0$, and the random jumps are modeled by the Poisson random measure $\int_{R^{+}} y J(t, d y)$.

We can specify different forms of Lévy measures to model different complex jump mechanisms. If we specify $\nu(d x)=\alpha x^{-1} e^{-\beta x} d x$ for small jumps in an infinitesimal time interval, then the Lévy subordinator in (1) is a temporally homogeneous gamma process (a gamma process with stationary increments) $G(t)$, with a density $f_{G(t)}=G a(x \mid \alpha t, \beta)=\frac{\beta^{\alpha t} x^{\alpha t-1} e^{-\beta x}}{\Gamma(\alpha t)}, x>0, t>$ 0. $G(t)$ has an infinite number of small jumps in a finite time interval, thus having infinite activity. The inverse Gaussian process has the similar feature, but with more very small jumps than $G(t)$, since its Lévy density $\nu(d x) / d x$ approaches to infinity faster than the gamma process does as $x$ goes to zero. Another case is the positive stable process whose probability distribution does not have a closed-form expression. For big jumps occurring according to the Poisson law, we can specify $\nu(d x)=\lambda \mu_{J}(d x)$, and then the Lévy subordinator is a compound Poisson process $C(t)$ with a jump density $\lambda$ and a jump size distribution $\mu_{J}$. $C(t)$ has a finite number of jumps over a 
finite time interval, i.e., finite activity. Another example of Lévy subordinators with finite activity is the negative binomial process. In the negative binomial process, however, the interarrival times of jumps are not exponentially distributed and the variance of the number of jumps in a time interval is larger than the corresponding mean [7].
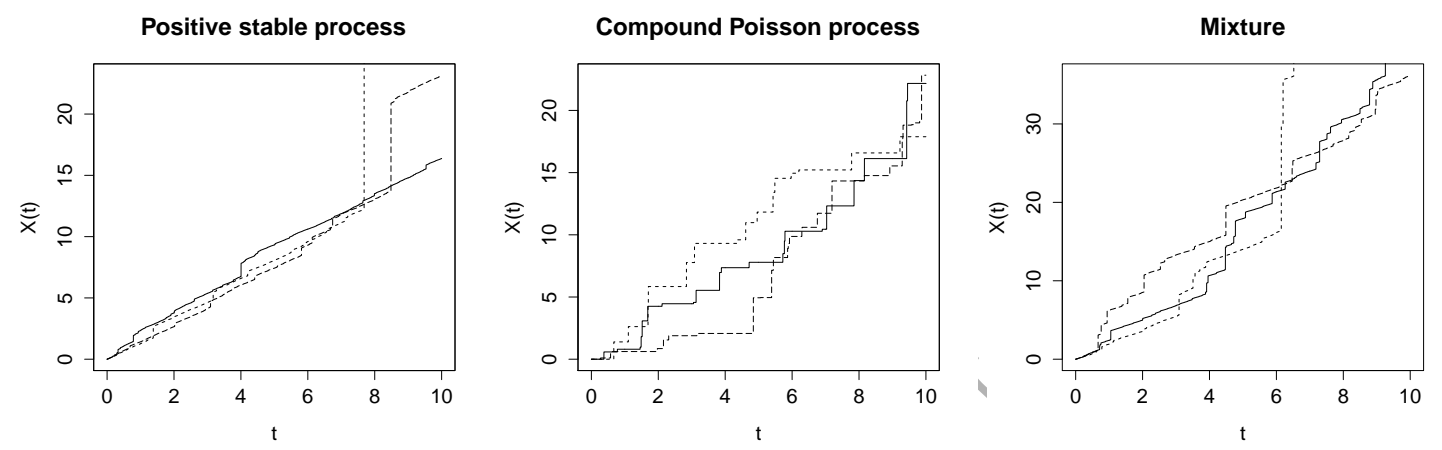

Figure 1: Sample paths of Lévy subordinators

To demonstrate Lévy subordinators, Figure 1 shows three sample paths of the positive stable process, the compound Poisson process, and the mixture of both, respectively, where the time interval length is $1 / 1000$. Notice that the increments of the positive stable process are due to consecutive infinite small jumps, while the increments of the compound Poisson process are due to sporadic jumps.

A system fails when the degradation process $X_{s}(t)$ exceeds a failure threshold $x$. Using $X_{s}(t)$, the lifetime of the system and its moments are defined respectively as $T_{x}=\inf \left\{t: X_{s}(t)>\right.$ $x\}, M\left(T_{X}^{m}, x\right)=E\left(T_{x}^{m}\right)$. The reliability function is defined as $R_{X}(x, t)=P\left(T_{x} \geq t\right)=$ $P\left(X_{s}(t) \leq x\right)=F_{X_{s}(t)}(x)$. The Laplace transform of $R_{X}(x, t)$ with respect to (w.r.t.) $t$ is defined to be $R_{X}^{L}(x, \omega)=\int_{R^{+}} e^{-\omega t} R_{X}(x, t) d t, \omega>0$. And the Laplace transform of $R_{X}^{L}(x, \omega)$ w.r.t. $x$ is $R_{X}^{L L}(u, \omega)=\int_{R^{+}} e^{-u x} R_{X}^{L}(x, \omega) d x, u>0$. The explicit expressions of $R_{X}(x, t)$ and 
lifetime moments $M\left(T_{X}^{m}, x\right)$ in terms of Laplace transform are

$$
\begin{aligned}
& R_{X}^{L L}(u, \omega)=u^{-1}\left\{\omega+b^{*} u-\int_{R^{+}}\left(e^{-u y}-1\right) \nu(d y)\right\}^{-1}, \\
& M^{L}\left(T_{X}^{m}, u\right)=m ! u^{-1}\left\{b^{*} u-\int_{R^{+}}\left(e^{-u y}-1\right) \nu(d y)\right\}^{-m},
\end{aligned}
$$

where $b^{*}=b-\int_{0<y<1} y \nu(d y)$ is a constant on $R^{+}$, and $\nu$ is the Lévy measure [31].

A non-Gaussian OU process $Y(t)$ is the solution of a stochastic differential equation driven by $X_{s}(t)$ :

$$
d Y(t)=\alpha Y(t) d t+d X_{s}(t) .
$$

The increment of $Y(t)$ depends on the current state, reflecting many degradation phenomena. As a functional extension of Lévy subordinator, the non-Gaussian OU process can be expressed as

$$
Y(t)=e^{\alpha t} Y(0)+\int_{0}^{t} e^{\alpha(t-\xi)} d X_{s}(\xi)
$$

$Y(0)$ represents the initial state of the degradation, and we assume $Y(0)=0$ as many new systems have not accumulated degradation when they are firstly operated. We assume $\alpha>0$, which guarantees that the degradation process is non-decreasing [31].

$$
\begin{aligned}
Y(t) & =\int_{0}^{t} e^{\alpha(t-\xi)} d X_{s}(\xi) \\
& =\int_{0}^{t} e^{\alpha(t-\xi)}\left(b d \xi+\int_{0<y<1} y(J(d \xi, d y)-\nu(d \xi, d y))+\int_{y \geq 1} y J(d \xi, d y)\right) \\
& =\frac{1}{\alpha}\left(e^{\alpha t}-1\right)\left(b-\int_{0<y<1} y \nu(d y)\right)+\int_{0}^{t} e^{\alpha(t-\xi)} \int_{R^{+}} y J(d \xi, d y) .
\end{aligned}
$$


In (5), the continuous degradation part is modeled by $\frac{1}{\alpha}\left(e^{\alpha t}-1\right)\left(b-\int_{0<y<1} y \nu(d y)\right)$, and the random jumps are modeled by the Poisson random measure $\int_{0}^{t} e^{\alpha(t-\xi)} \int_{R^{+}} y J(d \xi, d y)$. As illustrated in Figure 2, the mean degradation path of $Y(t)$ is exponential w.r.t $t$, while the mean path of $X_{s}(t)$ is linear shown in Figure 1.
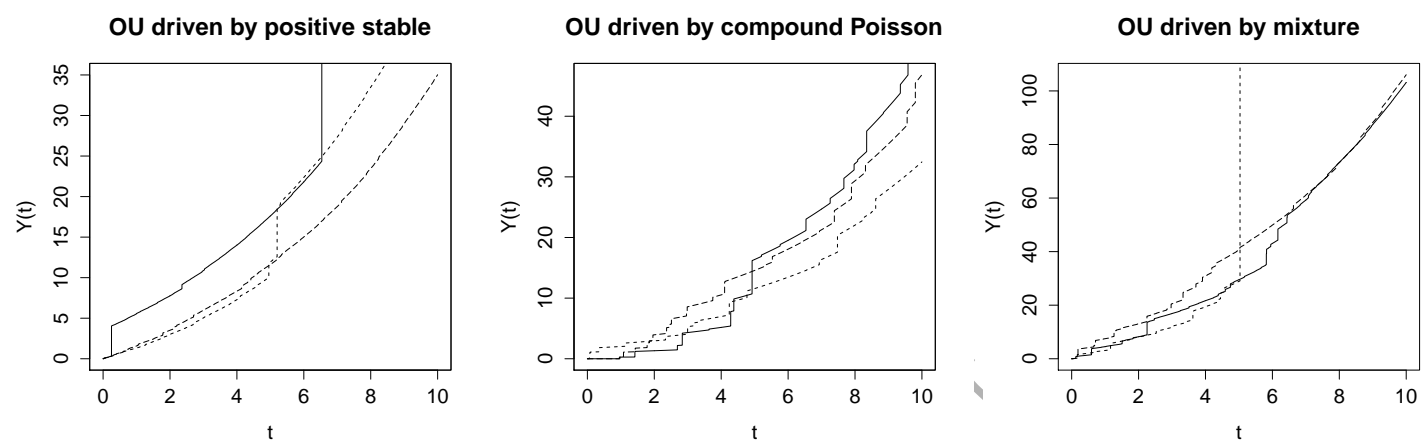

Figure 2: Sample paths of Lévy driven OU processes

Based on $Y(t)$, the definitions of lifetime, moments and reliability function are $T_{y}=\inf \{t$ : $Y(t)>y\}, M\left(T_{Y}^{m}, y\right)=E\left(T_{y}^{m}\right)$, and $R_{Y}(y, t)=P\left(T_{y} \geq t\right)=P(Y(t) \leq y)=F_{Y(t)}(y)$. The explicit expressions of $R_{Y}(y, t)$ and lifetime moments $M\left(T_{Y}^{m}, y\right)$ in terms of Laplace transform, represented by Lévy measures, are presented in (6) and (7). The detailed proofs are provided in [31].

$$
R_{Y}^{L L}(u, \omega)=-u^{-1} \int_{u}^{\infty} e^{F(v, u, \omega)} g(v) d v
$$

$$
M^{L}\left(T_{Y}^{m}, u\right)=(-1)^{m} u^{-1} m \alpha^{1-m} \int_{u}^{\infty}(\ln u-\ln v)^{m-1} e^{F(v, u)} g(v) d v
$$

where $F(v, u, \omega)=\int_{v}^{u} f\left(v^{\prime}, \omega\right) d v^{\prime}, f(v, \omega)=\left(\omega+b^{*} v-\int_{R^{+}}\left(e^{-v z}-1\right) \nu(d z)\right) / \alpha v, F(v, u)=$ $\int_{v}^{u} f\left(v^{\prime}\right) d v^{\prime}, f(v)=\left(b^{*} v-\int_{R^{+}}\left(e^{-v z}-1\right) \nu(d z)\right) / \alpha v$, and $g(v)=-1 / \alpha v$. In addition, $b^{*}=$ $b-\int_{0<z<1} z \nu(d z)$ is a constant on $R^{+}$, and $\nu$ is the Lévy measure. 


\section{Estimation}

The explicit formulae in (2) (3) (6) (7) provide a convenient tool to estimate the reliability function and lifetime moments for degradation processes with random jumps. In this section, we present the estimation methods for the parameters in $X_{s}(t)$ and $Y(t)$ using degradation-with-jump measures.

The probability density function of a general Lévy subordinator is not available ín a closedform. Thus the traditional maximum likelihood estimation and Bayesian estimation are not convenient for such general jump processes and their functional extensions. Based on the characteristic function of Lévy subordinator, we propose to use the cumulant M-estimator (CME) [25] and bootstrap method to construct the point estimates and confidence intervals for life characteristics.

\subsection{Estimation for Lévy Degradation Processes}

Based on Lévy Khintchine formula, a Lévy subordinator $X_{s}(t)$ has the characteristic function:

$$
\phi_{X_{s}(t)}(u)=E\left(e^{i u X_{s}(t)}\right)=e^{t \eta_{s}(u)}
$$

where

$$
\eta_{s}(u)=i b^{*} u+\int_{R^{+}}\left(e^{i u x}-1\right) \nu(d x)
$$

is the Lévy symbol.

\subsubsection{Point Estimates}

Due to the property of independent and identically distributed increments, the CME can achieve a consistent estimator using a single degradation path with enough data points. When multiple 
degradation paths are available from the same population, all the paths can be used to do estimation for more accurate results.

A degradation path can be discretized as $\vec{x}_{n}=\left(x_{1 \Delta}, x_{2 \Delta}, \ldots, x_{n \Delta}\right)$, where $\Delta$ is the step of the discretely measured data series. We denote $\Theta$ as the parameter vector in $\eta_{s}(u)$, i.e., $\eta_{s}(u) \equiv$ $\eta_{s}(u ; \Theta), \phi_{X_{s}(1)}(u) \equiv \phi_{X_{s}(1)}(u ; \Theta)$. The Lévy symbol $\eta_{s}(u)=\log \phi_{X_{s}(1)}(u)$, is also called the cumulant function.

We choose a preliminary estimator $\hat{\phi}_{X_{s}(\Delta)}\left(u ; \vec{x}_{n}\right)$, either almost surely

$$
\hat{\phi}_{X_{s}(\Delta)}\left(u ; \vec{x}_{n}\right) \stackrel{a . s .}{\longrightarrow} \phi_{X_{s}(\Delta)}(u ; \widehat{\Theta})
$$

or in probability

$$
\hat{\phi}_{X_{s}(\Delta)}\left(u ; \vec{x}_{n}\right) \stackrel{P}{\longrightarrow} \phi_{X_{s}(\Delta)}(u ; \Theta)
$$

as $n \rightarrow \infty$.

Define $w()$ as an integrable weight function with compact support. $w()$ is symmetric around the origin and is strictly positive on a neighbourhood of the origin. For example, $w(u)=I_{u \in[-l, l]}, l>$ 0 , or $w(u)=e^{-u^{2}}$.

The space of square integrable functions w.r.t $w$ is

$$
f^{2}(w)=\left\{f: R \rightarrow C \mid f \text { is measurable and } \int|f(u)|^{2} w(u) d u<\infty\right\}
$$

where $C$ is the complex space. 
The semi-inner product $\left(f_{1}, f_{2}\right)_{w}$ on $\digamma^{2}(w)$ is defined as

$$
\left(f_{1}, f_{2}\right)_{w}=\Re \int f_{1}(u) \overline{f_{2}(u)} w(u) d u
$$

where $\overline{f_{2}(u)}$ is the complex conjugate of $f_{2}(u)$, and $\Re f$ is the real part of $f$. The semi-norm on $\digamma^{2}(w)$ is defined as $\|f\|_{w}=(f, f)_{w}^{\frac{1}{2}}$.

The CME is

$$
\hat{\Theta}_{n}=\underset{\Theta}{\operatorname{argmin}} \mathfrak{D}\left(\Theta ; \vec{x}_{n}\right)
$$

where $\mathfrak{D}\left(\Theta ; \vec{x}_{n}\right)$ is the weighted difference between cumulants:

$$
\begin{aligned}
\mathfrak{D}\left(\Theta ; \vec{x}_{n}\right) & =\left\|\log \hat{\phi}_{X_{s}(\Delta)}\left(u ; \vec{x}_{n}\right)-\log \phi_{X_{s}(\Delta)}(u ; \Theta)\right\|_{w}^{2} \\
& =\int\left|\log \hat{\phi}_{X_{s}(\Delta)}\left(u ; \vec{x}_{n}\right)-\log \phi_{X_{s}(\Delta)}(u ; \Theta)\right|^{2} w(u) d u \\
& =\int \Delta^{2}\left|\hat{\eta}_{s}\left(u ; \vec{x}_{n}\right)-\eta_{s}(u ; \Theta)\right|^{2} w(u) d u .
\end{aligned}
$$

With $\hat{\Theta}_{n}$, the point estimators of reliability function and lifetime moments in (2) and (3), $\hat{R}_{X}(x, t)$ and $\hat{M}\left(T_{X}^{m}, x\right)$, can be obtained by inverting $\hat{R}_{X}^{L L}\left(u, \omega ; \hat{\Theta}_{n}\right)$ and $\hat{M}^{L}\left(T_{X}^{m}, u ; \hat{\Theta}_{n}\right)$, respectively.

When we have multipe sample paths $\vec{x}_{n_{j}}^{j}=\left(x_{1 \Delta}^{j}, x_{2 \Delta}^{j}, \ldots, x_{n_{j} \Delta}^{j}\right), j \in\{1,2, \cdots, M\}$, the CME is

$$
\hat{\Theta}_{\sum_{j=1}^{M} n_{j}}=\underset{\Theta}{\operatorname{argmin}} \mathfrak{D}\left(\Theta ; \vec{x}_{n_{1}}^{1}, \vec{x}_{n_{2}}^{2}, \cdots, \vec{x}_{n_{M}}^{M}\right)
$$

where $\mathfrak{D}\left(\Theta ; \vec{x}_{n_{1}}^{1}, \vec{x}_{n_{2}}^{2}, \cdots, \vec{x}_{n_{M}}^{M}\right)=\left\|\log \hat{\phi}_{X_{s}(\Delta)}\left(u ; \vec{x}_{n_{1}}^{1}, \vec{x}_{n_{2}}^{2}, \cdots, \vec{x}_{n_{M}}^{M}\right)-\log \phi_{X_{s}(\Delta)}(u ; \Theta)\right\|_{w}^{2}$. 


\subsubsection{Bootstrap Confidence Intervals}

We construct confidence intervals for $R_{X}(x, t)$ and $M\left(T_{X}^{m}, x\right)$ based on bootstrap simulation with the following steps:

1. Obtain $\hat{\Theta}_{n}$ or $\hat{\Theta}_{\sum_{j=1}^{M} n_{j}}$ by using the CME for one or $M$ sample paths in (8) or (9). The sample paths can be the real data in practice or the simulated data from $X_{s}(t ; \Theta)$ by setting an initial value for $\Theta$.

2. Generate one sample path $\overrightarrow{\ddot{x}}_{n}=\left(\ddot{x}_{1 \Delta}, \ddot{x}_{2 \Delta}, \ldots, \ddot{x}_{n \Delta}\right)$ from $X_{s}\left(t ; \hat{\Theta}_{n}\right)$, or $M$ sample paths $\overrightarrow{\ddot{x}}_{n_{j}}^{j}=\left(\ddot{x}_{1 \Delta}^{j}, \ddot{x}_{2 \Delta}^{j}, \ldots, \ddot{x}_{n_{j} \Delta}^{j}\right), j \in\{1,2, \cdots, M\}$ from $X_{s}(t ; \hat{\Theta}$

3. Get the bootstrap estimates $\hat{\ddot{\Theta}}_{n}$ or $\hat{\Theta}_{\sum_{j=1}^{M} n_{j}}$ using the CME, based on $\overrightarrow{\ddot{x}}_{n}=\left(\ddot{x}_{1 \Delta}, \ddot{x}_{2 \Delta}, \ldots, \ddot{x}_{n \Delta}\right)$ or $\ddot{\ddot{x}}_{n_{j}}^{j}=\left(\ddot{x}_{1 \Delta}^{j}, \ddot{x}_{2 \Delta}^{j}, \ldots, \ddot{x}_{n_{j} \Delta}^{j}\right), j \in\{1,2, \cdots, M\}$.

4. Obtain the bootstrap estimates $\hat{\ddot{R}}_{X}^{L L}\left(u, \omega ; \hat{\ddot{\Theta}}_{n}\right)$ and $\hat{\ddot{M}}^{L}\left(T_{X}^{m}, u ; \hat{\ddot{\Theta}}_{n}\right)$, or $\hat{\ddot{R}}_{X}^{L L}\left(u, \omega ; \hat{\ddot{\Theta}}_{j=1}^{M} n_{j}\right)$ and $\hat{\ddot{M}}^{L}\left(T_{X}^{m}, u ; \hat{\ddot{\Theta}}_{\sum_{j=1}^{M} n_{j}}\right)$.

5. Repeat Steps $2-4 K$ times to obtain $\hat{\vec{R}}_{X ; k}^{L L}\left(u, \omega ; \hat{\Theta}_{n}\right)$ and $\hat{\ddot{M}}_{k}^{L}\left(T T_{X}^{m}, u ; \hat{\Theta}_{n}\right)$, or $\hat{\ddot{R}}_{X ; k}^{L L}\left(u, \omega ; \hat{\Theta}_{\sum_{j=1}^{M} n_{j}}\right)$ and $\hat{\ddot{M}}_{k}^{L}\left(T_{X}^{m}, u ; \hat{\Theta}_{\sum_{j=1}^{M} n_{j}}\right), 1 \leq k \leq K$.

6. Implement the inversion algorithm for Laplace transform to invert $\hat{\ddot{R}}_{X ; k}^{L L}$ and $\hat{\ddot{M}}_{k}^{L}$, obtaining $\hat{\ddot{R}}_{X ; k}(x, t)$ and $\hat{\ddot{M}}_{k}\left(T_{X}^{m}, x\right), 1 \leq k \leq K$.

7. Sort $\hat{\ddot{R}}_{X ; k}(x, t), 1 \leq k \leq K$ in ascending order for each $x$ and $t$, obtaining $\hat{\ddot{R}}_{X ;[k]}(x, t), 1 \leq$ $k \leq K$. Sort $\hat{\ddot{M}}_{k}\left(T_{X}^{m}, x\right), 1 \leq k \leq K$ in ascending order for each $x$, obtaining $\hat{\ddot{M}}_{[k]}\left(T_{X}^{m}, x\right), 1 \leq k \leq K$.

8. Compute the $100(1-\delta) \%$ confidence intervals for $R_{X}(x, t):\left[\hat{\ddot{R}}_{X ;[l]}(x, t), \hat{\ddot{R}}_{X ;[u]}(x, t)\right]$ and 
for $M\left(T_{X}^{m}, x\right): \quad\left[\hat{\ddot{M}}_{[i]}\left(T_{X}^{m}, x\right), \hat{\ddot{M}}_{[\dot{u}]}\left(T_{X}^{m}, x\right)\right]$, where $i=\Phi\left(2 \Phi^{-1}(\chi)+\Phi^{-1}(\delta / 2)\right) K, \dot{u}=$ $\Phi\left(2 \Phi^{-1}(\chi)+\Phi^{-1}(1-\delta / 2)\right) K$, for $R_{X}(x, t)$

$$
\chi=\frac{\sum_{k} I_{\hat{\vec{R}}_{X ; k}(x, t) \leq \hat{R}_{X}(x, t)}}{K},
$$

for $M\left(T_{X}^{m}, x\right)$,

$$
\chi=\frac{\sum_{k} I_{\hat{\ddot{M}}_{k}\left(T_{X}^{m}, x\right) \leq \hat{M}\left(T_{X}^{m}, x\right)}}{K},
$$

and $\Phi$ is the standard normal distribution function used in [26]. $\hat{\ddot{R}}_{X ;[i]}(x, t)$ and $\hat{\ddot{R}}_{X ;[\dot{u}]}(x, t)$ are approximate pointwise lower and upper one-sided $100(1-\delta / 2) \%$ biasedcorrected confidence bounds for $R_{X}(x, t)$; and $\hat{\ddot{M}}_{[i]}\left(T_{X}^{m}, x\right)$ and $\hat{\ddot{M}}_{[\dot{u}]}\left(T_{X}^{m}, x\right)$ are approximate pointwise lower and upper one-sided $100(1-\delta / 2) \%$ biased-corrected confidence bounds for $M\left(T_{X}^{m}, x\right)[17]$

\subsection{Estimation for OU Degradation Processes}

\subsubsection{Point Estimates}

An OU degradation path can be discretized as $\vec{y}_{n}=\left(y_{1 \Delta}, y_{2 \Delta}, \ldots, y_{n \Delta}\right)$, where $\Delta$ is the step of the discretely measured data series. Since the OU process driven by a Lévy subordinator has dependent increments, the CME cannot be directly used to estimate the parameters $(\alpha, \Theta)$ in $Y(t)$. Three steps are proposed to obtain the point estimates of $(\alpha, \Theta)$ : (1) estimate $\alpha,(2)$ estimate the increments of the background driving process, Lévy subordinator, using the estimator of $\alpha$ [11], and (3) the CME is activated to estimate $\Theta$ using the estimated increments. When multipe OU degradation paths are available from the same population, all the paths can be used to do estimation following the same procedure. 
The discrete OU process can be expressed as

$$
Y_{i \Delta}=e^{\alpha \Delta} Y_{(i-1) \Delta}+\int_{(i-1) \Delta}^{i \Delta} e^{\alpha(i \Delta-\xi)} d X_{s}(\xi), 1 \leq i \leq n
$$

which is an analogue of the discrete-time first-order autoregression processes (AR(1)) with nonnegative innovations:

$$
Y_{i \Delta}=\rho Y_{(i-1) \Delta}+Z_{i \Delta}, 1 \leq i \leq n,
$$

by setting $\rho=e^{\alpha \Delta}>1$, and $Z_{i \Delta}=\int_{(i-1) \Delta}^{i \Delta} e^{\alpha(i \Delta-\xi)} d X_{s}(\xi)$. Since $X_{s}(t)$ is a Lévy subordinator, $Z_{i \Delta}$ is nonnegatively independent and identically distributed. Taking the advantage of the nonnegativity of the increments of the background driving process, Lévy subordinator, we choose the following linear programming estimator for $\rho$ :

$$
\hat{\rho}_{n}=\min _{1 \leq i \leq n} y_{i \Delta} / y_{(i-1) \Delta}
$$

The estimator for $\alpha$ is

$$
\hat{\alpha}_{n}=\log \hat{\rho}_{n} / \Delta
$$

Assuming $\rho>0$ and the distribution function $F$ of $Z_{i \Delta}$ is regularly varying at zero with exponent $\vartheta$, (i.e., there exists $\vartheta>0$ such that $\lim _{a \rightarrow 0} \frac{F(a x)}{F(x)}=x^{\vartheta}, x>0$ ), [16] showed that $\hat{\rho}_{n} \stackrel{a . s .}{\longrightarrow}$ $\rho$ and developed the asymptotic distributions for both stationary and nonstationary cases. This estimator has been further studied for the stationary case of autoregressive processes (when $0<$ $\rho<1$ ) [11]. In another way, $\hat{\rho}_{n}$ can be viewed as the solution to the linear programming problem of maximizing the objective function $g(\rho)=\rho$ subject to $n$ linear constraints $y_{i \Delta}-\rho y_{(i-1) \Delta} \geq 0,1 \leq$ 
$i \leq n$, and therefore, it is called the linear programming estimator $[19,20] . \hat{\rho}_{n}$ is equal to the maximum likelihood estimator conditioned on $Y_{0}$ if $Z_{i \Delta}$ is exponentially distributed. [27] showed that $\hat{\rho}_{n}$ is strongly consistent for a broad range of $F$, including both light-tailed and heavy-tailed distributions.

Based on (4), the increment of the Lévy subordinator is

$$
X_{i \Delta}-X_{(i-1) \Delta}=Y_{i \Delta}-Y_{(i-1) \Delta}-\alpha \int_{(i-1) \Delta}^{i \Delta} Y(\xi) d \xi
$$

The estimated increments by the trapezoidal approximation for the integral can be valued as

$$
x_{i \Delta}-x_{(i-1) \Delta}=y_{i \Delta}-y_{(i-1) \Delta}-\hat{\alpha}_{n} \Delta \frac{y_{i \Delta}+y_{(i-1) \Delta}}{2}, 1 \leq i \leq n .
$$

Then we can estimate $\Theta$ in $X_{s}(t)$ by the CME in (8) using $x_{i \Delta}-x_{(i-1) \Delta}, 1 \leq i \leq n$. With $\hat{\alpha}_{n}$ and $\hat{\Theta}_{n}$, the point estimators of reliability function and lifetime moments in (6) and (7) are $\hat{R}_{Y}(y, t)$ and $\hat{M}\left(T_{Y}^{m}, y\right)$ by inverting $\hat{R}_{Y}^{L L}\left(u, \omega ; \hat{\alpha}_{n}, \hat{\Theta}_{n}\right)$ and $\hat{M}^{L}\left(T_{Y}^{m}, u ; \hat{\alpha}_{n}, \hat{\Theta}_{n}\right)$, respectively.

When we have multiple sample paths $\vec{y}_{n_{j}}^{j}=\left(y_{1 \Delta}^{j}, y_{2 \Delta}^{j}, \ldots, y_{n_{j} \Delta}^{j}\right), j \in\{1,2, \cdots, M\}$, we have:

$$
\hat{\alpha}_{\sum_{j=1}^{M} n_{j}}=\frac{\log \left(\min _{\substack{1 \leq j \leq M \\ 1 \leq i \leq n_{j}}} y_{i \Delta}^{j} / y_{(i-1) \Delta}^{j}\right)}{\Delta},
$$

and $\Theta$ in $X_{s}(t)$ can be estimated by the CME in (9). 


\subsubsection{Bootstrap Confidence Intervals}

We construct confidence intervals for $R_{Y}(y, t)$ and $M\left(T_{Y}^{m}, y\right)$ based on bootstrap simulation. The steps are:

1. Obtain $\hat{\alpha}_{n}$ or $\hat{\alpha}_{\sum_{j=1}^{M} n_{j}}$ using the linear programming estimator for one or $M$ sample paths in (10) or (11). The sample paths can be the real data in practice or the simulated data from $Y(t ; \alpha, \Theta)$ by setting a value for $(\alpha, \Theta)$.

2. Estimate the increments of the background driving Lévy subordinator, obtaining $\hat{\Theta}_{n}$ or $\hat{\Theta}_{\sum_{j=1}^{M} n_{j}}$ using the CME in (8) or (9).

3. Generate one sample path $\vec{y}_{n}=\left(\ddot{y}_{1 \Delta}, \ddot{y}_{2 \Delta}, \ldots, \ddot{y}_{n \Delta}\right)$ from $Y\left(t ; \hat{\alpha}_{n}, \hat{\Theta}_{n}\right)$ or $M$ sample paths $\overrightarrow{\ddot{y}}_{n_{j}}^{j}=\left(\ddot{y}_{1 \Delta}^{j}, \ddot{y}_{2 \Delta}^{j}, \ldots, \ddot{y}_{n_{j} \Delta}^{j}\right), j \in\{1,2, \cdots, M\}$ from $Y\left(t ; \hat{\alpha}_{\sum_{j=1}^{M} n_{j}}, \hat{\Theta}_{\sum_{j=1}^{M} n_{j}}\right)$.

4. Obtain the bootstrap estimates $\left(\hat{\ddot{\alpha}}_{n}, \hat{\ddot{\Theta}}_{n}\right)$ or $\left(\hat{\ddot{\alpha}}_{\sum_{j=1}^{M} n_{j}}, \hat{\Theta}_{\sum_{j=1}^{M} n_{j}}\right)$ using the CME, based on $\vec{y}_{n}=\left(\ddot{y}_{1 \Delta}, \ddot{y}_{2 \Delta}, \ldots, \ddot{y}_{n \Delta}\right)$ or $\vec{y}_{n_{j}}^{j}=\left(\ddot{y}_{1 \Delta}^{j}, \ddot{y}_{2 \Delta}^{j}, \ldots, \ddot{y}_{n_{j} \Delta}^{j}\right), j \in\{1,2, \cdots, M\}$.

5. Get the bootstrap estimates $\hat{\ddot{R}}_{Y}^{L L}\left(u, \omega ; \hat{\ddot{\alpha}}_{n}, \hat{\ddot{\Theta}}_{n}\right)$ and $\hat{\ddot{M}}^{L}\left(T_{Y}^{m}, u ; \hat{\ddot{\alpha}}_{n}, \hat{\Theta}_{n}\right)$, or $\hat{\ddot{R}}_{Y}^{L L}\left(u, \omega ; \hat{\ddot{\alpha}}_{\sum_{j=1}^{M} n_{j}}, \hat{\ddot{\Theta}}_{\sum_{j=1}^{M} n_{j}}\right)$ and $\hat{\ddot{M}}^{L}\left(T_{Y}^{m}, u ; \hat{\ddot{\alpha}}_{\sum_{j=1}^{M} n_{j}}, \hat{\ddot{\Theta}}_{\sum_{j=1}^{M} n_{j}}\right)$.

6. Repeat Steps $3-5 K$ times to obtain $\hat{\ddot{R}}_{Y ; k}^{L L}\left(u, \omega ; \hat{\ddot{\alpha}}_{n}, \hat{\ddot{\Theta}}_{n}\right)$ and $\hat{\ddot{M}}_{k}^{L}\left(T_{Y}^{m}, u ; \hat{\ddot{\alpha}}_{n}, \hat{\Theta}_{n}\right)$, or $\hat{\ddot{R}}_{Y, k}^{L L}\left(u, \omega ; \hat{\ddot{\alpha}}_{\sum_{j=1}^{M} n_{j}}, \hat{\ddot{\Theta}}_{\sum_{j=1}^{M} n_{j}}\right)$ and $\hat{\ddot{M}}_{k}^{L}\left(T_{Y}^{m}, u ; \hat{\ddot{\alpha}}_{\sum_{j=1}^{M} n_{j}}, \hat{\ddot{\Theta}}_{\sum_{j=1}^{M} n_{j}}\right), 1 \leq k \leq K$.

7. Implement the inversion algorithm for Laplace transform to invert $\hat{\ddot{R}}_{Y ; k}^{L L}$ and $\hat{\ddot{M}}_{k}^{L}$, obtaining $\hat{\ddot{R}}_{Y ; k}(y, t)$ and $\hat{\ddot{M}}_{k}\left(T_{Y}^{m}, y\right), 1 \leq k \leq K$.

8. Sort $\hat{\ddot{R}}_{Y ; k}(y, t), 1 \leq k \leq K$ in ascending order for each $y$ and $t$, obtaining $\hat{\ddot{R}}_{Y ; k]}(y, t), 1 \leq$ $k \leq K$. Sort $\hat{\ddot{M}}_{k}\left(T_{Y}^{m}, y\right), 1 \leq k \leq K$ in ascending order for each $y$, obtaining $\hat{\ddot{M}}_{[k]}\left(T_{Y}^{m}, y\right), 1 \leq k \leq K$. 
9. Compute the $100(1-\delta) \%$ confidence intervals for $R_{Y}(y, t):\left[\hat{\ddot{R}}_{Y ;[i]}(y, t), \hat{\ddot{R}}_{Y ; i u]}(y, t)\right]$ and for $M\left(T_{Y}^{m}, y\right):\left[\hat{\ddot{M}}_{[i]}\left(T_{Y}^{m}, y\right), \hat{\ddot{M}}_{[\dot{u}]}\left(T_{Y}^{m}, y\right)\right]$, where $i=\Phi\left(2 \Phi^{-1}(\chi)+\Phi^{-1}(\delta / 2)\right) K, \dot{u}=$ $\Phi\left(2 \Phi^{-1}(\chi)+\Phi^{-1}(1-\delta / 2)\right) K$, for $R_{Y}(y, t)$

$$
\chi=\frac{\sum_{k} I_{\hat{\tilde{R}}_{Y ; k}(y, t) \leq \hat{R}_{Y}(y, t)}}{K},
$$

for $M\left(T_{Y}^{m}, y\right)$,

$$
\chi=\frac{\sum_{k} I_{\hat{\bar{M}}_{k}\left(T_{Y}^{m}, y\right) \leq \hat{M}\left(T_{Y}^{m}, y\right)}}{K} .
$$

$\hat{\ddot{R}}_{Y ;[i]}(y, t)$ and $\hat{\ddot{R}}_{Y ;[i]}(y, t)$ are approximate pointwise lower and upper one-sided 100(1 $\delta / 2) \%$ biased-corrected confidence bounds for $R_{Y}(y, t)$; and $\hat{\ddot{M}}_{[i]}\left(T_{Y}^{m}, y\right)$ and $\hat{\ddot{M}}_{[i]}\left(T_{Y}^{m}, y\right)$ are approximate pointwise lower and upper one-sided $100(1-\delta / 2) \%$ biased-corrected confidence bounds for $M\left(T_{Y}^{m}, y\right)$.

\section{Simulation Study}

To illustrate our proposed method, we use an interesting Lévy measure

$$
\nu(d x)=\frac{\kappa}{\Gamma(1-\kappa)} \frac{1}{x^{\kappa+1}} d x
$$

where $x>0,0<\kappa<1$, which represents a positive stable process $P S(\kappa)$ whose distribution is in general unknown in closed-form [7]. Notice that if $\kappa$ is close to 0 , the process propagates with big jumps; and if $\kappa$ is close to 1 , the process evolves with small jumps. The distribution of this variable is asymmetry and heavy-tailed, i.e., it does not have moments of order $\kappa$ and above. 
Proposition 1. The characteristic function of $P S(\kappa)$ is

$$
\phi_{X}(u)=\exp \left(-t \cos \frac{\pi \kappa}{2}|u|^{\kappa}\left(1-i \tan \frac{\pi \kappa}{2} \operatorname{sgn}(u)\right)\right)
$$

where $\operatorname{sgn}(u)$ is the sign function.

Proof.

$$
\begin{aligned}
& \eta_{s}(u)=\int_{R^{+}}\left(e^{i u x}-1\right) \frac{\kappa}{\Gamma(1-\kappa)} \frac{1}{x^{\kappa+1}} d x=\frac{\kappa}{\Gamma(1-\kappa)} \int_{R^{+}} x^{-\kappa} d x\left(-x^{-1}+x^{-1} e^{i u x}\right) \\
& =\frac{\kappa}{\Gamma(1-\kappa)} \int_{R^{+}} x^{-\kappa} d x \int_{-i u}^{0} e^{-y x} d y=\frac{\kappa}{\Gamma(1-\kappa)} \int_{-i u}^{0} d y \int_{R^{+}} x^{-\kappa} e^{-y x} d x \\
& =\kappa \int_{-i u}^{0} y^{\kappa-1} d y=-(-i u)^{\kappa}= \begin{cases}-(-u)^{\kappa} e^{\frac{i \pi \kappa}{2}} & u<0 \\
-u^{\kappa} e^{\frac{-i \pi \kappa}{2}} & u>0\end{cases} \\
& =\left\{\begin{array}{l}
-(-u)^{\kappa}\left(\cos \frac{\pi \kappa}{2}+i \sin \frac{\pi \kappa}{2}\right) \quad u<0 \\
-u^{\kappa}\left(\cos \frac{\pi \kappa}{2}-i \sin \frac{\pi \kappa}{2}\right) \quad u>0
\end{array}\right. \\
& =-\cos \frac{\pi \kappa}{2}|u|^{\kappa}\left(1-i \tan \frac{\pi \kappa}{2} \operatorname{sgn}(u)\right)
\end{aligned}
$$

Remark 1. $P S(\kappa)$ is a class of general stable processes $S(\kappa, \beta, \gamma, \delta)$, where $\kappa \in(0,2]$ is the index parameter, $\beta \in[-1,1]$ is the skewness parameter, $\gamma>0$ is the scale parameter, and $\delta$ is the shift parameter. The Lévy symbol of $S(\kappa, \beta, \gamma, \delta)$ is $\eta_{s}(u)=-\gamma^{\kappa}|u|^{\kappa}\left(1-i \beta \tan \frac{\pi \kappa}{2} \operatorname{sgnu}\right)+i u \delta$. Thus by setting $0<\kappa<1, \beta=1, \gamma^{\kappa}=\cos \frac{\pi \kappa}{2}=\left|1-i \tan \frac{\pi \kappa}{2}\right|^{-1}$, and $\delta=0$, we get $P S(\kappa)$.

In the simulation study, one degradation path is used to illustrate the proposed procedure that can be also applied for multiple degradation paths. We choose $\Delta=1$ without losing the generality. 
The empirical characteristic function $\hat{\phi}_{X_{s}(1)}\left(u ; \vec{x}_{n}\right)=\frac{1}{n} \sum_{i=1}^{n} e^{i u\left(x_{i}-x_{i-1}\right)}$ is used as the preliminary estimator in (8). The CME of $\kappa$ is

$$
\hat{\kappa}=\underset{\kappa}{\operatorname{argmin}}|| \log \left(\frac{1}{n} \sum_{i=1}^{n} e^{i u\left(x_{i}-x_{i-1}\right)}\right)-\left.\frac{-\left(1-i \tan \frac{\pi \kappa}{2} \operatorname{sgnu}\right)}{\left|1-i \tan \frac{\pi \kappa}{2}\right|}|u|^{\kappa}\right|_{w} ^{2},
$$

where $w(u)=I_{u \in[-L, L]}$ for $L>0$.

Table 1: Results of $\hat{\kappa}$ for $P S(\kappa)$

\begin{tabular}{ccc}
\hline $\mathbf{n}$ & mean & mean squared error \\
\hline 20 & 0.8912641 & 0.003706321 \\
50 & 0.9029252 & 0.001141654 \\
100 & 0.8987184 & 0.0007026813 \\
\hline
\end{tabular}

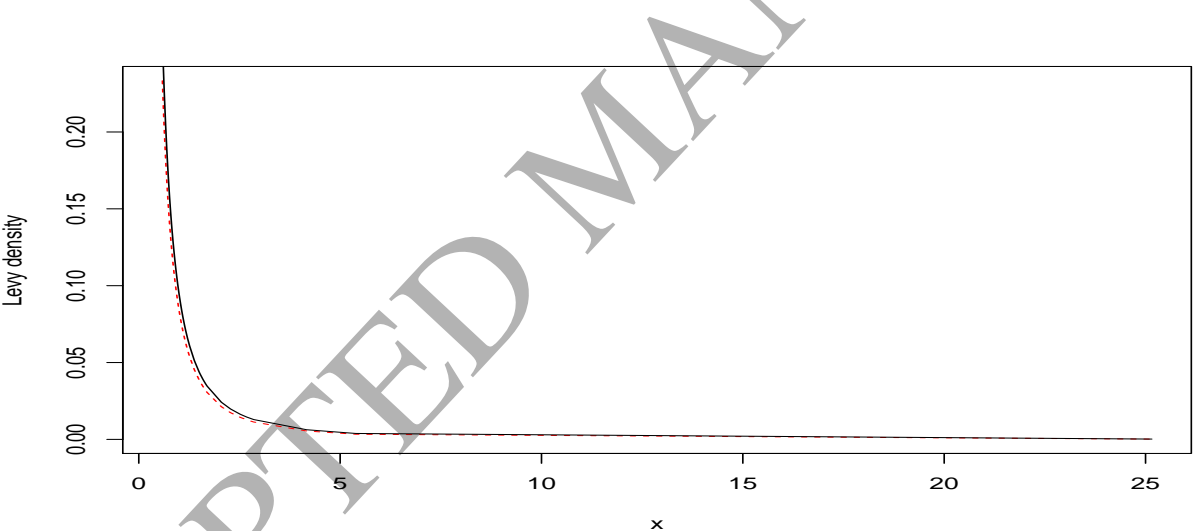

Figure 3: Lévy density of $P S(\kappa)$; dashed line: estimated values when $\mathrm{n}=25$; solid line: true values

We simulate one path with $n$ data points from $P S(\kappa)$ by setting $\kappa=0.9$, and perform estimation to obtain $\hat{\kappa}$. The optimization problem is solved numerically using a quasi-Newton method. We repeat the estimation 1000 times to calculate the mean and the mean squared error (MSE) of $\hat{\kappa}$. Table 1 shows the results of $\hat{\kappa}$ for $P S(\kappa)$ based on the simulated data. The mean is closed to 0.9 and the MSE is small. Figure 3 shows the estimated Lévy density, compared with the true Lévy density of $P S(\kappa)$. 
Proposition 2. The characteristic function of the OU process driven by $P S(\kappa)$ is

$$
\phi_{Y}(u)=-(\alpha \kappa)^{-1}\left(e^{\alpha \kappa t}-1\right) \cos \frac{\pi \kappa}{2}|u|^{\kappa}\left(1-i \tan \frac{\pi \kappa}{2} \operatorname{sgn}(u)\right) .
$$

Proof. For the OU process driven by a general Lévy subordinator, the characteristic function can be expressed as

$$
E\left[e^{i u Y(t)}\right]=\exp \left\{\int_{0}^{t}\left[i b^{*} u e^{\alpha r}+\int_{R^{+}}\left(e^{i u e^{\alpha r} y}-1\right) \nu(d y)\right] d r\right\} .
$$

Then for the OU process driven by $P S(\kappa)$,

$$
\begin{aligned}
\phi_{Y}(u) & =\exp \left\{\int_{0}^{t} \int_{R^{+}}\left(e^{i u e^{\alpha r} x}-1\right) \frac{\kappa}{\Gamma(1-\kappa)} \frac{1}{x^{\kappa+1}} d r\right\} \\
& =\exp \left\{\int_{0}^{t}-\cos \frac{\pi \kappa}{2}\left|u e^{\alpha r}\right| \kappa\left(1-i \tan \frac{\pi \kappa}{2} \operatorname{sgn}\left(u e^{\alpha r}\right)\right) d r\right\} \\
& =-\cos \frac{\pi \kappa}{2}\left(1-i \tan \frac{\pi \kappa}{2} \operatorname{sgn}(u)\right)|u|^{\kappa} \int_{0}^{t} e^{\alpha r \kappa} d r \\
& =-(\alpha \kappa)^{-1}\left(e^{\alpha \kappa t}-1\right) \cos \frac{\pi \kappa}{2}|u|^{\kappa}\left(1-i \tan \frac{\pi \kappa}{2} \operatorname{sgn}(u)\right) .
\end{aligned}
$$

The simulation study is performed using the procedures in Section 3.2. We simulate one path with $n$ data points from the OU process driven by $P S(\kappa)$, by setting $\alpha=0.1, \kappa=0.9$, and perform estimation to obtain $\hat{\alpha}$ and $\hat{\kappa}$. The estimation is repeated 1000 times to calculate the mean and the mean squared error (MSE) of $\hat{\alpha}$ and $\hat{\kappa}$, respectively. Table 2 shows the results of $\hat{\alpha}$ and $\hat{\kappa}$ for the OU driven by $P S(\kappa)$ based on the simulated data. Figure 4 shows the estimated increments compared with the true increments of $P S(\kappa)$. Figure 5 shows the estimated Lévy density compared with the 
true Lévy density of OU driven by $P S(\kappa)$.

Table 2: Results of $\hat{\alpha}$ and $\hat{\kappa}$ for the OU driven by $P S(\kappa)$

\begin{tabular}{c|cc|cc}
\hline & $\hat{\alpha}$ & & $\hat{\kappa}$ & \\
\hline $\mathbf{n}$ & mean & mean squared error & mean & mean squared error \\
\hline 20 & 0.1101515 & 0.0001279552 & 0.8915346 & 0.006616674 \\
50 & 0.1087836 & $9.227509 \mathrm{e}-05$ & 0.9078137 & 0.001910659 \\
100 & 0.1081567 & $7.684354 \mathrm{e}-05$ & 0.9060823 & 0.001126384 \\
\hline
\end{tabular}

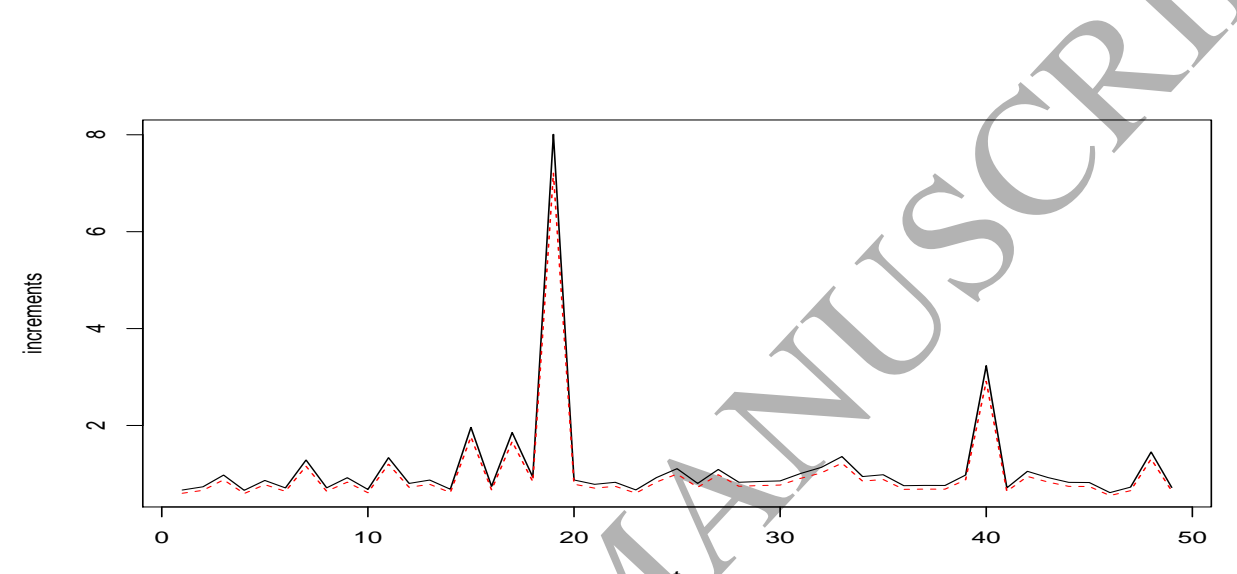

Figure 4: Increments of the background driving $P S(\kappa)$; dashed line: estimated values when $\mathrm{n}=50$; solid line: true values

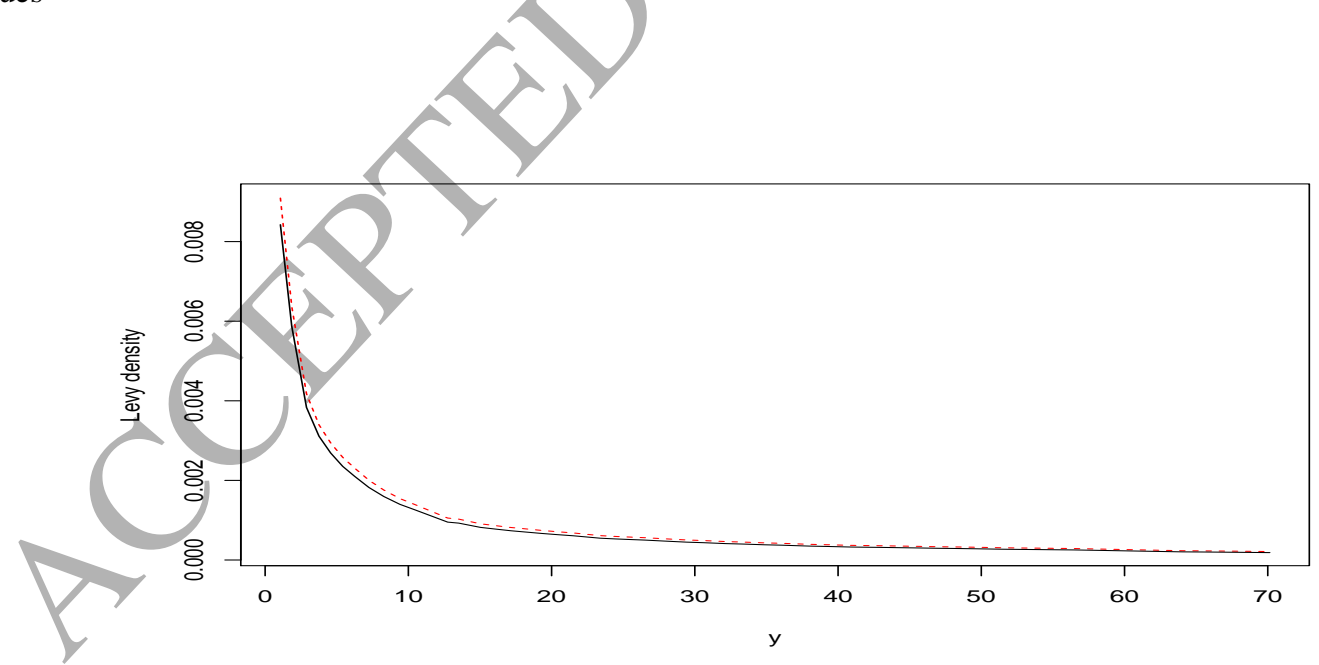

Figure 5: Marginal Lévy density of OU driven by $P S(\kappa)$; dashed line: estimated values when $\mathrm{n}=50$; solid line: true values

When the degradation evolution can be described by this positive stable process, the Laplace 
expression of reliability function based on (2) is

$$
R_{X}^{L L}(u, \omega)=u^{-1}\left\{\omega+u^{\kappa}\right\}^{-1}
$$

Based on (3), the Laplace expression of lifetime moments is

$$
M^{L}\left(T_{X}^{m}, u\right)=m ! u^{-m \kappa-1}
$$

When the evolution of the degradation can be described by the non-Gaussian OU process driven by $P S(\kappa)$, the Laplace expression of reliability function based on (6), is

$$
R_{Y}^{L L}(u, \omega)=\alpha^{-1} u^{\alpha^{-1} \omega-1} e^{\alpha^{-1} \frac{1}{\kappa} u^{\kappa}} \int_{u}^{\infty} v^{\mathcal{Y}\left(\alpha^{-1} \omega+1\right)} e^{-\alpha^{-1} \frac{1}{\kappa} v^{\kappa}} d v
$$

Based on (7), the Laplace expression of lifetime moments is

$$
M^{L}\left(T_{Y}^{m}, u\right)=u^{-1} m \alpha{ }^{-m} \sum_{a=0}^{m-1} C_{m-1}^{a}(-1)^{a}(\ln u)^{a} e^{\alpha^{-1} \frac{1}{\kappa} u^{\kappa}} \int_{u}^{\infty}(\ln v)^{m-1-a} v^{-1} e^{-\alpha^{-1} \frac{1}{\kappa} v^{\kappa}} d v .
$$

The inversion algorithms for Laplace transform $[1,10]$ were implemented to invert Laplace expressions in order to compute the values of reliability and lifetime moments. Figure 6 and Figure 7 show the confidence intervals for reliability function at a given failure threshold based on the bootstrap simulation, in which the sample size is set to be 500 . Besides the Lévy measure used in this example, we can specify different Lévy measures to fit the corresponding degradation data, in order to analyze reliability and lifetime characteristics. 


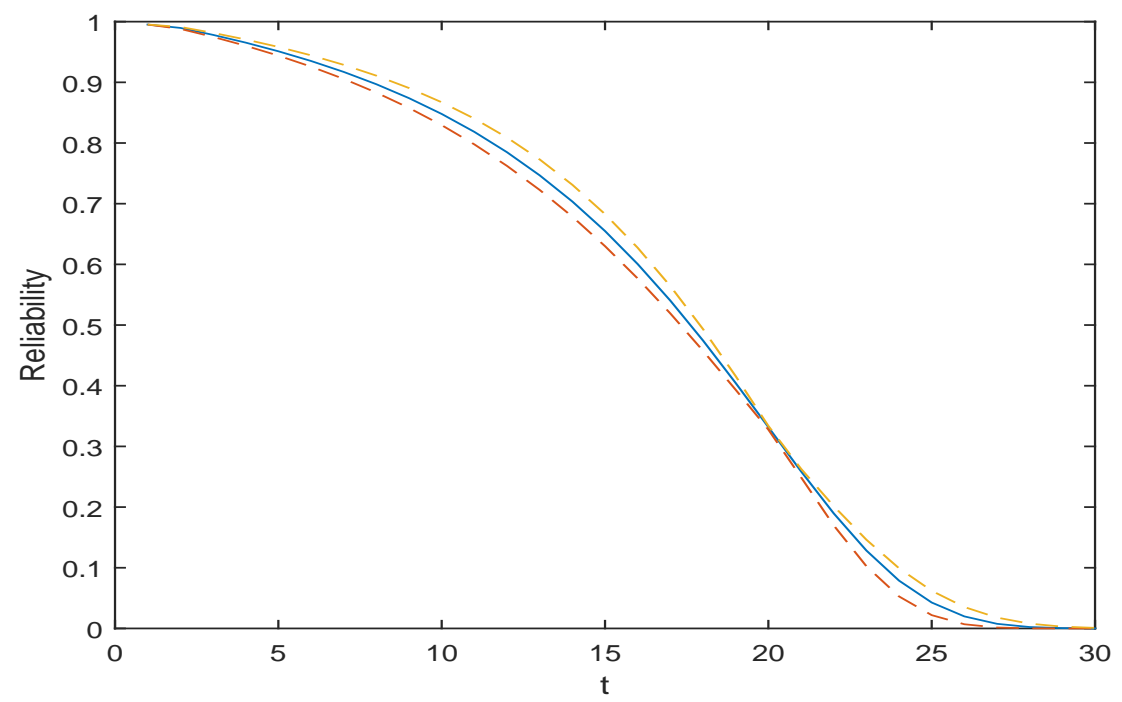

Figure 6: $90 \%$ confidence interval of reliability function for $X_{s}(t)$

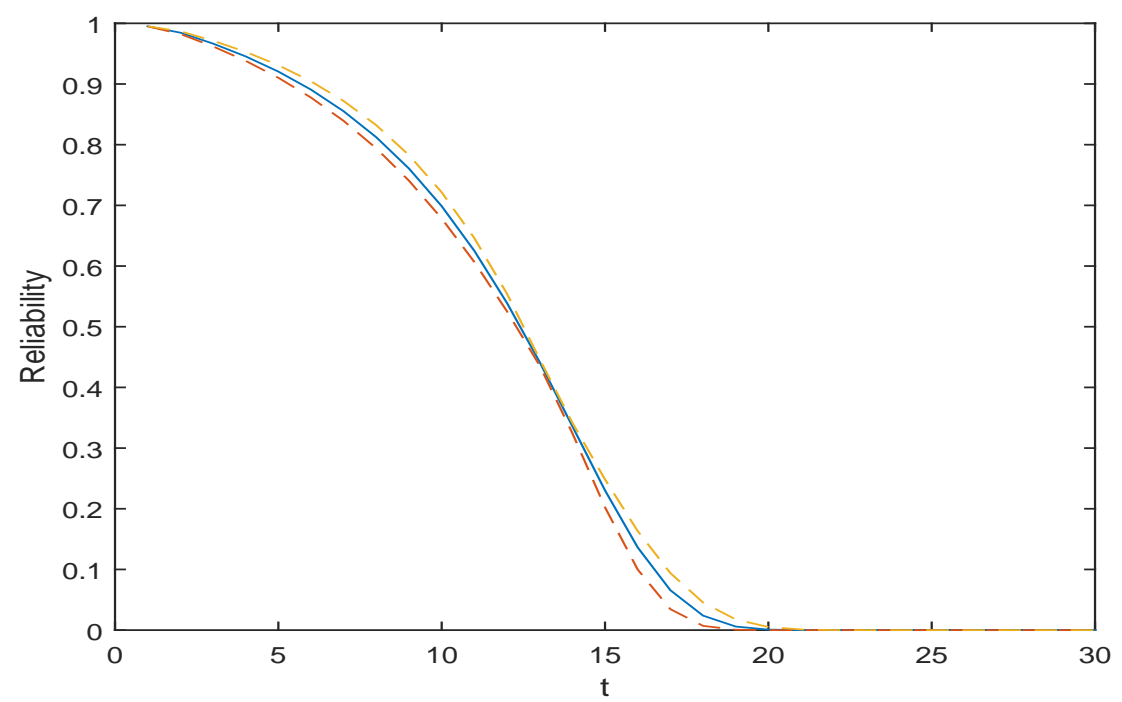

Figure 7: $90 \%$ confidence interval of reliability function for $Y(t)$

\section{Case Study}

In this section, the proposed method is applied to analyze the degradation data of lithium-ion batteries from the randomized battery usage test [8]. We choose the capacity data of four 18650 
lithium-ion batteries, which were tested under the room temperature with random charging and discharging current and time (Figure 8). Statistical test is performed for the presence of jumps using the bipower variation [6]. The p-value is close to zero, which indicates that there are significant jumps in the capacity loss processes of such batteries. In other words, a jump process is suitable to fit these degradation data series.

\section{Degradation of Li-ion Battery}

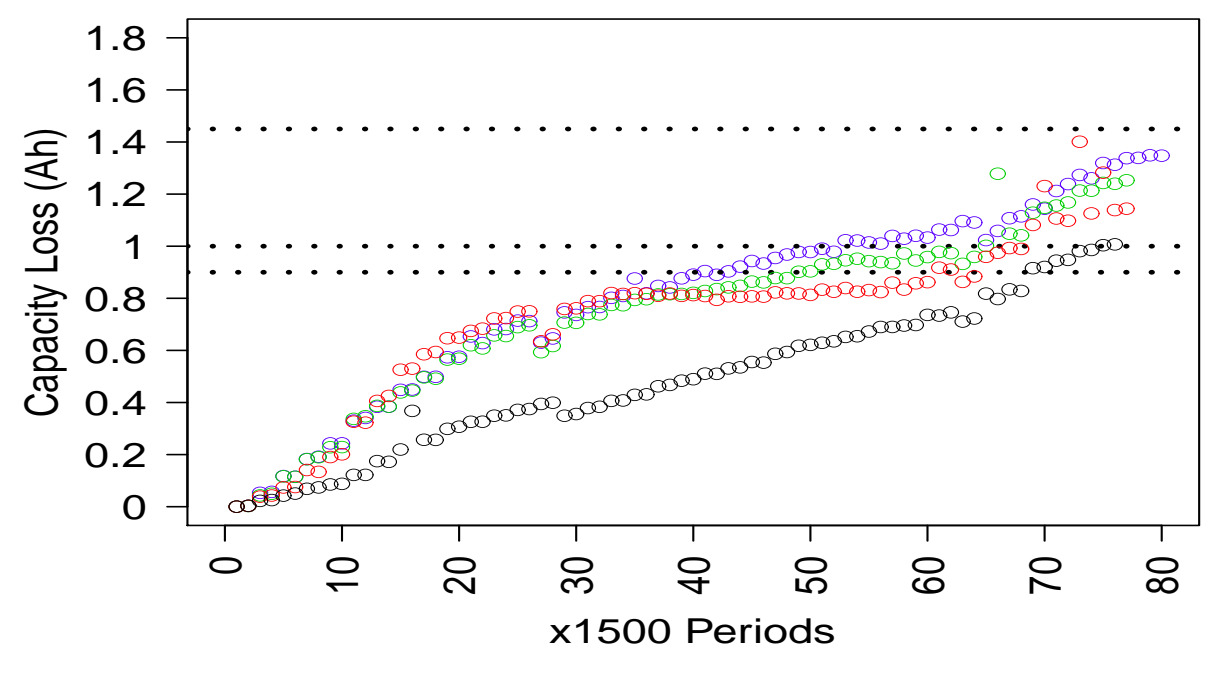

Figure 8: The capacity losing processes of four $18650 \mathrm{Li}$-ion batteries

We use a general Lévy process to fit the data that appear to have a linear trend. The corresponding Lévy measure is

$$
\nu(d y)=\frac{\delta \gamma^{-2 \kappa} \kappa y^{-\kappa-1} \exp \left(-\frac{1}{2} \gamma^{2} y\right)}{\Gamma(\kappa) \Gamma(1-\kappa)} d y
$$

which can cover: (1) the positive tempered stable process, when $y, \delta>0,0<\kappa<1, \gamma \geq 0$; (2) the positive stable process, when $y, \delta>0,0<\kappa<1, \gamma=0$; (3) the inverse Gaussian process, when $y, \delta>0,0<\kappa<1, \gamma=0.5$; and (4) the gamma process, when $y, \delta>0, \kappa \rightarrow 0, \gamma>0$. 
Using the CME in (9), we obtain $\hat{\delta}=2.9884776, \hat{\gamma}=2.0335391$ and $\hat{\kappa}=0.1511678$. The Laplace expression of reliability estimator based on (2) is

$$
\hat{R}^{L L}(u, \omega)=u^{-1}\left\{\omega-\hat{\delta} \hat{\gamma}+\hat{\delta}\left(\hat{\gamma}^{\frac{1}{\hat{\kappa}}}+2 u\right)^{\hat{\kappa}}\right\}^{-1}
$$

Based on (3), the Laplace expression of lifetime moments' estimator is

$$
\left.\hat{M}^{L}\left(T^{n}, u\right)=n ! u^{-1}\left\{-\hat{\delta} \hat{\gamma}+\hat{\delta}\left(\hat{\gamma}^{\frac{1}{\hat{\kappa}}}+2 u\right)^{\hat{\kappa}}\right\}\right\}^{n}
$$
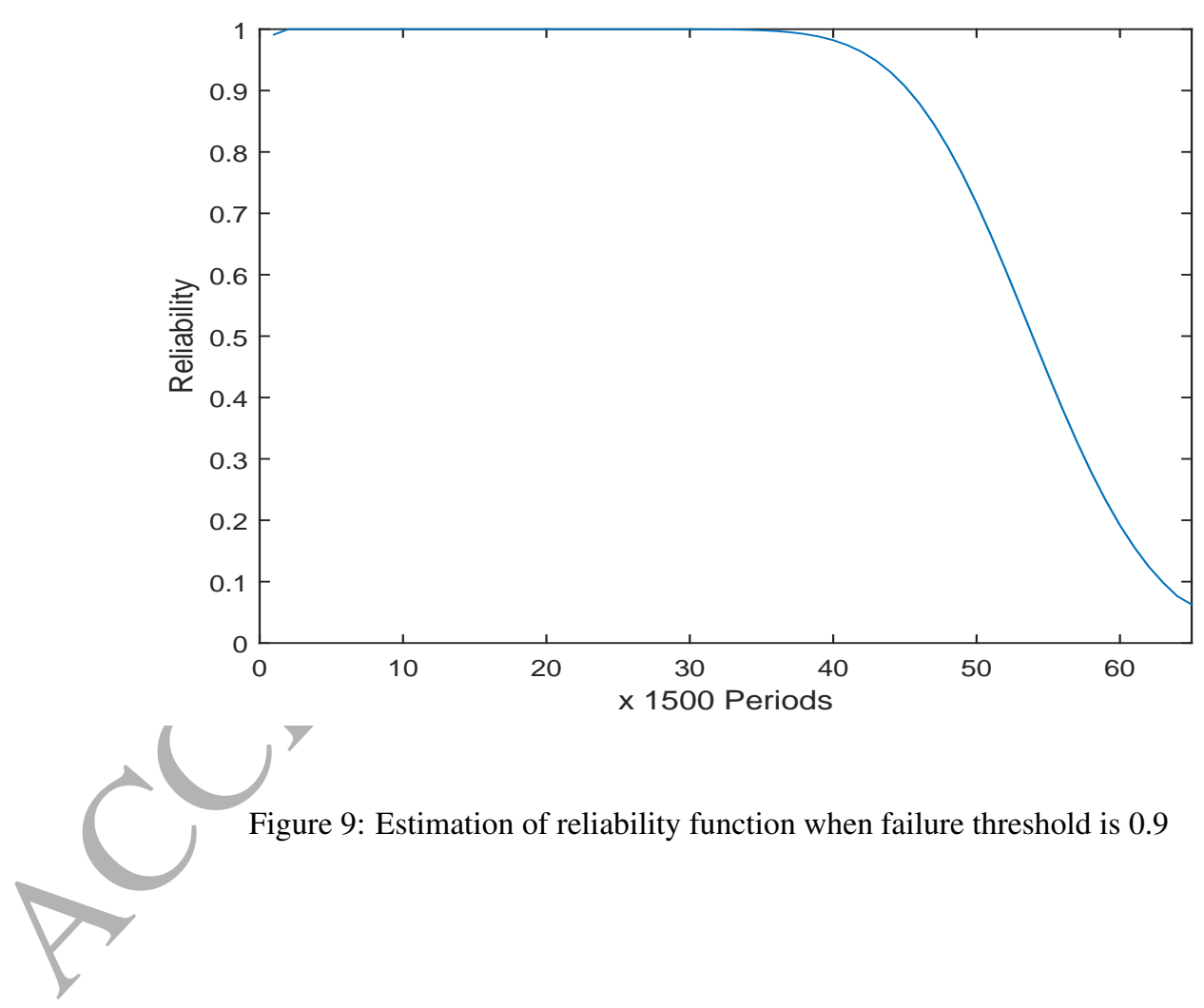

Figure 9: Estimation of reliability function when failure threshold is 0.9 


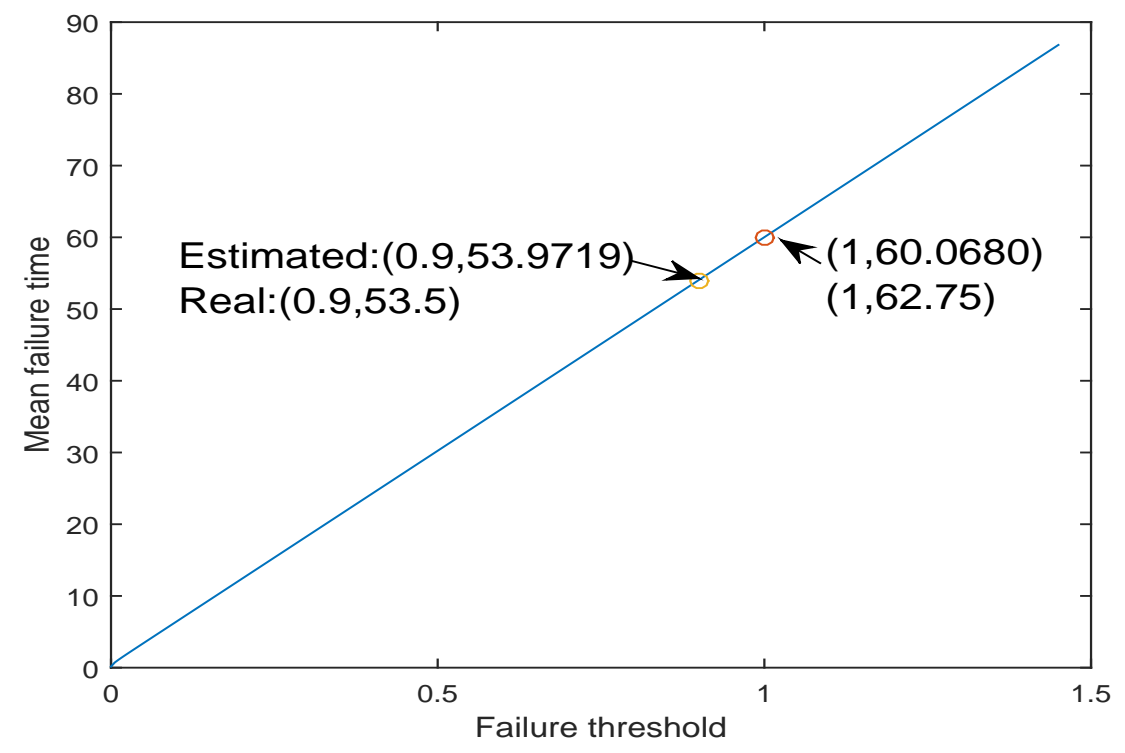

Figure 10: Estimation of first moments of lifetime w.r.t. failure threshold

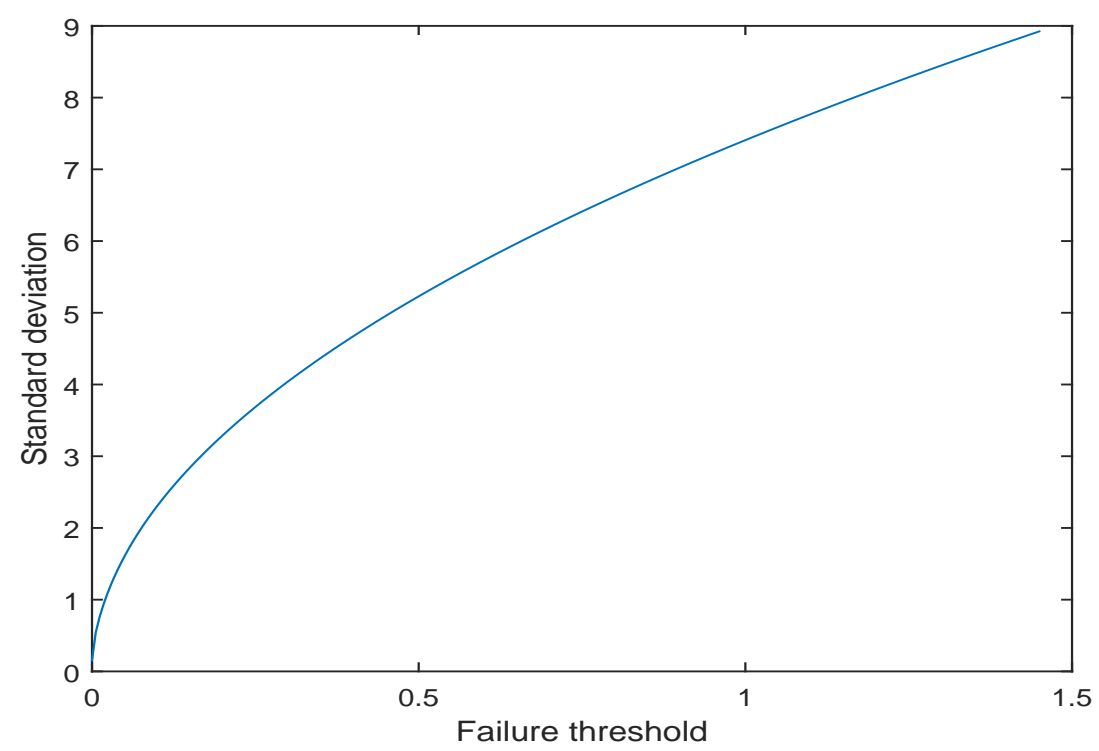

Figure 11: Estimation of standard deviation of lifetime w.r.t. failure threshold 


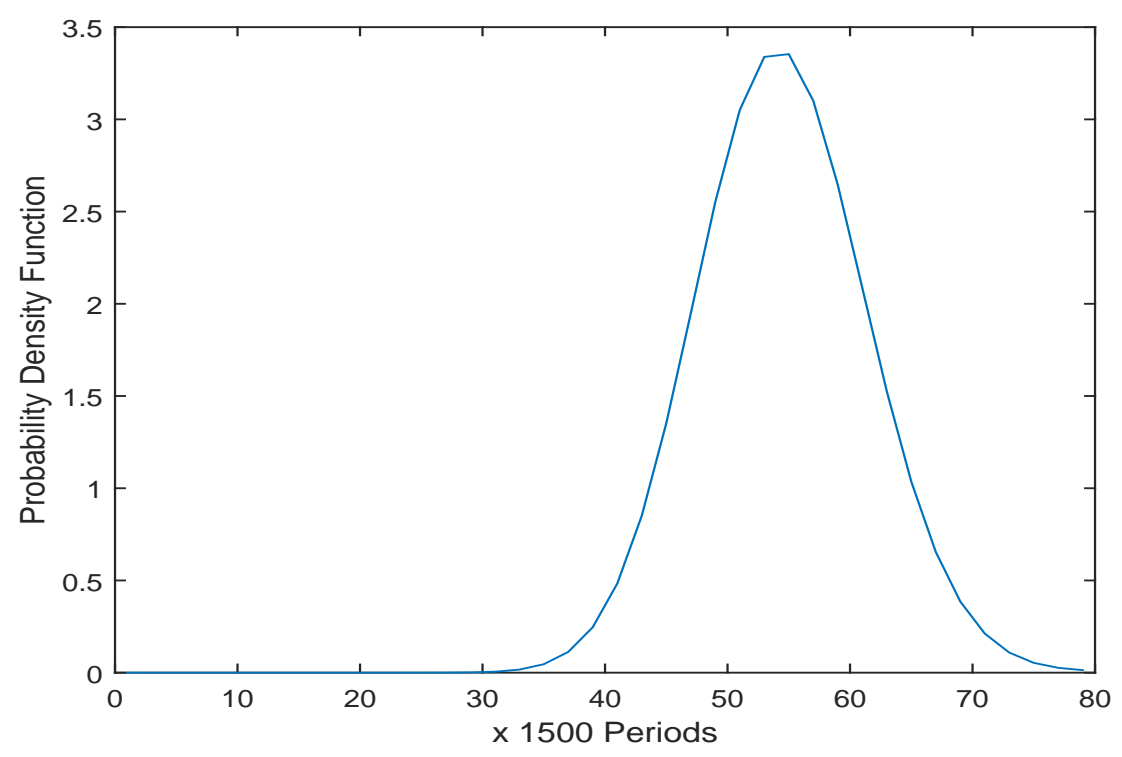

Figure 12: Estimation of probability density function when failure threshold is 0.9

\section{Degradation of Li-ion Battery}

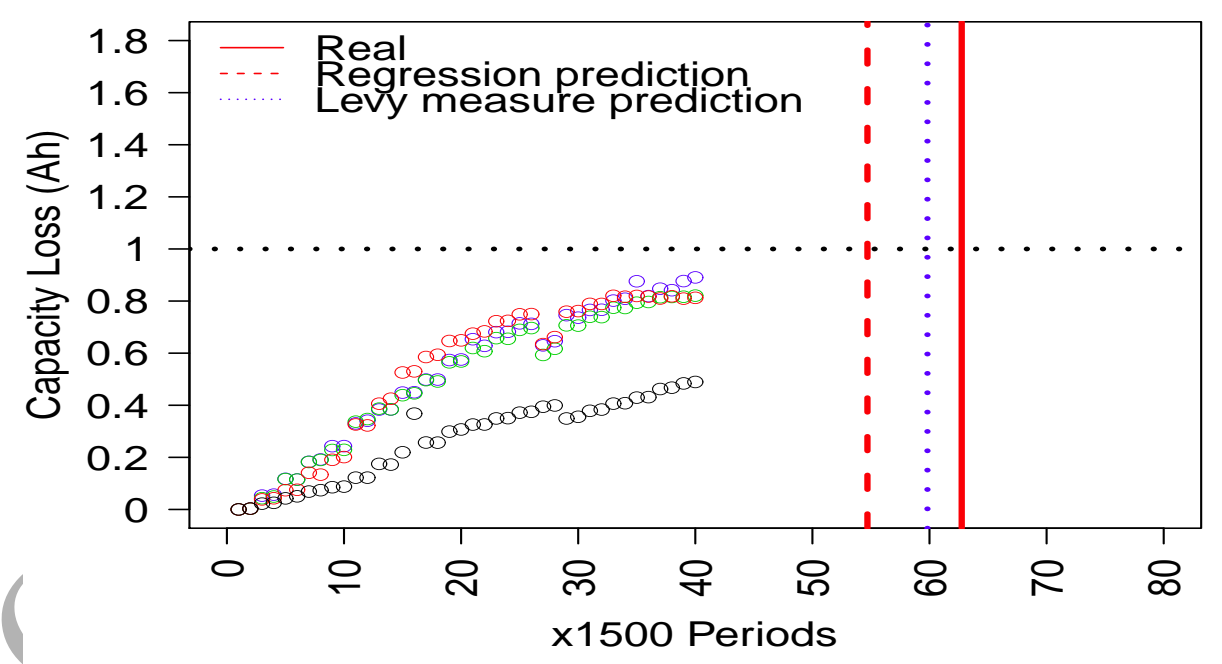

Figure 13: Comparison result between our method and a regression method without considering the jumps on the mean failure time prediction

Figure 9 and 12 show the estimation of reliability function and pdf for the Li-ion batteries, 
assuming the failure threshold is 0.9, respectively. In Figure 10, the solid line is the estimated mean failure time w.r.t. the failure threshold; when the failure threshold is 0.9 , the estimated mean failure time is 53.9719 , while the average of failure times of four batteries is 53.5 ; when the failure threshold is 1 , the estimated mean failure time is 60.068 , while the the average of failure times of four batteries is 62.75 . Figure 11 illustrates the estimation of standard deviation of failure time w.r.t. the failure threshold. Figure 13 shows the comparison result between our method and a regression method without considering the jumps on the mean failure time prediction, based on the first 40 data points. The Lévy based prediction is more accurate.

\section{Conclusions}

In this paper, we construct general stochastic models to integrally handle uncertainties and jumps using Lévy and non-Gaussian OU processes. Our model can fit a great deal of degradation data with jumps (e.g., linear/nonlinear, light/heavy-tailed). We developed systematic procedures for estimating reliability characteristics based on the CME and bootstrap simulation. Our proposed method provides explicit results for precise reliability analysis.

Lévy processes with non-monotonic paths are suitable to model degradation processes in systems that have self-healing properties and/or undergo random maintenance actions. These Lévy processes may contain Gaussian part and/or negative jumps with Lévy measures defined on the whole $R$ domain, due to which the first passage time of such processes is analytically intractable. Subsequently, the reliability analysis based on such non-monotonic Lévy processes is interesting and challenging to be explored.

\section{Acknowledgements}


This paper is based upon work supported by the Texas Norman Hackerman Advanced Research Program under Grant no. 003652-0122-2009, and by USA National Science Foundation (NSF) under Grant no. 0970140 and Grant no. 1728321.

\section{References}

[1] J. Abate and W. Whitt, Numerical inversion of Laplace transforms of probability distributions, ORSA J. Comput. 1995, 7, 36-43.

[2] D. Applebaum, Lévy processes and stochastic calculus, 2nd ed., Cambridge University Press, Cambridge, 2009.

[3] O.E. Barndorff-Nielsen and N. Shephard, Non-Gaussian Ornstein-Uhlenbeck-based models and some of their uses in financial economics, Journal of the Royal Statistical Society, Series B, Statistical Methodology 2001, 63 (2), 167-207.

[4] O.E. Barndorff-Nielsen and N. Shephard, Econometric analysis of realized volatility and its use in estimating stochastic volatility models, Journal of the Royal Statistical Society, Series B, Statistical Methodology 2002, 64 (2), 253-280.

[5] O.E. Barndorff-Nielsen and N. Shephard, Integrated OU processes and non-Gaussian OUbased stochastic volatility models, Scandinavian Journal of Statistics 2003, 30, 277-295.

[6] O.E. Barndorff-Nielsen and N. Shephard, Econometrics of testing for jumps in financial economics using bipower variation, Journal of Financial Econometrics, 2006 4(1), 1-30.

[7] O.E. Barndorff-Nielsen and N. Shephard, Basics of Lévy processes, Unpublished chapter of the authors' book on Lévy Driven Volatility Models, 2012. 
[8] B. Bole, C. Kulkarni, and M. Daigle, Randomized battery usage data set, NASA Ames Prognostics Data Repository, 2014.

[9] M. Boulanger and L.A. Escobar, Experimental design for a class of accelerated degradation tests, Technometrics 1994, 36(3), 260-272.

[10] L. Brančík, Numerical inversion of two-dimensional Laplace transforms based on partial inversions, Proceedings of 17th International Conference Radioelektronika 2007, Brno, Czech Republic, 451-454.

[11] P.J. Brockwell, R.A. Davis and Y. Yang, Estimation for nonnegative Lévy-driven OrnsteinUhlenbeck Processes, Journal of Applied Probability 2007, 44 (4), 977-989.

[12] A. Brouste, M. Fukasawa, H. Hino, S.M. Iacus, K. Kamatani, Y. Koike, H. Masuda, R. Nomura, T. Ogihara, Y. Shimuzu, M. Uchida, and N. Yoshida, The YUIMA project: A computational framework for simulation and inference of stochastic differential equations, Journal of Statistical Software, 2014, 57(4), 1-51.

[13] N. Chen, and K. Tsui, Condition monitoring and remaining useful life prediction using degradation signals: Revisited. IIE Transactions 2013, 45(9), 939-952.

[14] E. Çinlar, Shock and wear models and Markov additive processes, in: I.N. Shimi, C.P. Tsokos (Eds.), The Theory and Applications of Reliability, Academic Press, New York, 1977, 193214.

[15] R. Cont and P. Tankov, Financial modelling with jump processes, Chapman \& Hall, New York, 2004. 
[16] R.A. Davis and W.P. McCormick, Estimation for first-order autoregressive processes with positive or bounded innovations, Stochastic Processes and their Applications 1989, 31(2), 237-250.

[17] B. Efron and R. J. Tibshirani, An Introduction to the Bootstrap, Chapman \& Hall 1993.

[18] J. D. Esary, A. W. Marshall, and F. Proshan, Shock models and wear processes, The Annals of Probability 1973, 1, 627-649.

[19] P.D. Feigin and S.I. Resnick, Limit distributions for linear programming time series estimators, Stochastic Processes and their Applications 1994, 51(1), 135-165.

[20] P.D. Feigin and S.I. Resnick, Linear programming estimators and bootstrapping for heavy tailed phenomena, Advances in Applied Probability 1997, 29(3), 759-805.

[21] M.P.S. Gander and D.A. Stephens, Simulation and inference for stochastic volatility models driven by Lévy processes, Biometrika 2007, 94 (3), 627-646.

[22] J.E. Griffin, M.F.J. Steel, Inference with non-Gaussian Ornstein-Uhlenbeck processes for stochastic volatility,J. Econometics 2006, 134, 605-644.

[23] K. Hasilová, and D. Vališ, Non-parametric estimates of the first hitting time of Li-ion battery, Measurement, 2018, 113, 82-91.

[24] G. Jin, and D. Matthews, Reliability demonstration for long-life products based on degradation testing and a Wiener process model. IEEE Transactions on Reliability 2014, 63(3), 781-797. 
[25] G. Jongbloed and F.H. van der Meulen, Parametric estimation for subordinators and induced OU processes, Scandinavian Journal of Statistics, 2006, 33(4), 825-847.

[26] C.J. Lu and W.Q. Meeker, Using degradation measures to estimate a time-to-failure distribution, Technometrics 1993, 35(2), 161-174.

[27] D. Preve, Linear programming-based estimators in nonnegative autoregression, Journal of Banking \& Finance 2015, 61(2), S225-S234.

[28] G.O. Roberts, O. Papaspiliopoulos, and P. Dellaportas, Bayesian inference for non-Gaussian Ornstein-Uhlenbeck stochastic volatility processes. J. Roy. Statist. Soc. Ser. B Statist. Methodol. 2004, 66(2), 369-393.

[29] K. Sato, Lévy processes and infinitely divisible distributions, Cambridge University Press, Cambridge, 1999.

[30] Y. Shu, Q. Feng, and D.W. Coit, Life distribution analysis based on Lévy subordinators for degradation with random jumps, Naval Research Logistics 2015, 62(6):483-492.

[31] Y. Shu, Q. Feng, E. Kao, and H. Liu, Lévy driven non-Gaussian Ornstein-Uhlenbeck processes for degradation-based reliability analysis, IIE Transactions 2016, 48(11): 9931003.

[32] K. Sobczyk, Stochastic models for fatigue damage of materials, Advances in Applied Probability 1987, 19, 652-673.

[33] E. Taufer and N. Leonenkob, Characteristic function estimation of non-Gaussian 
OrnsteinUhlenbeck processes, Journal of Statistical Planning and Inference 2009, 139, 30503063.

[34] E. Taufer, N. Leonenko,Simulation of Lévy-driven Ornstein-Uhlenbeck processes with given marginal distribution, Computational Statistics and Data Analysis 2009, 53, 2427-2437.

[35] D. Vališs, L. Žák, and O. Pokora, Perspective approach in using anti-oxidation and anti-wear particles from oil to estimate residual technical life of a system, Tribology International, 2018, 46-59.

[36] D. Vališ, O. Nováček, K. Hasilová, and Jan Leuchter, Modelling of degradation and a soft failure moment during the operation of a supercapacitor applying selected diffusion processes, Engineering Failure Analysis, 2017, 82, 566-582.

[37] D. Vališ, and D. Mazurkiewicz, Application of selected Levy processes for degradation modelling of long range mine belt using real-time data, Archives of Civil and Mechanical Engineering, 2018, 18 (4), 1430-1440.

[38] D. Vališ, L. Žák, O. Pokora, and P. Lánský, Perspective analysis outcomes of selected tribodiagnostic data used as input for condition based maintenance, Reliability Engineering and System Safety 2016, 145(1), 231-242.

[39] J.M. Van Noortwijk, A survey of the application of gamma processes in maintenance, Reliability Engineering and System Safety 2009, 94, 2-21.

[40] X. Wang and D. Xu, An inverse Gaussian process model for degradation data, Technometrics 2010, 52(2), 188-197. 
[41] Z. Wang, Y. Zhang, Q. Wu, H. Fu, C. Liu, and S. Krishnaswamy, Degradation reliability modeling based on an independent increment process with quadratic variance. Mechanical Systems and Signal Processing 2016, 70-71, 467-483.

[42] G.A. Whitmore, Estimating degradation by a Wiener diffusion process subject to measurement error, Lifetime Data Analysis 1995, 1, 307-319.

[43] G.A. Whitmore, M.J. Crowder, and J.F. Lawless, Failure inference from a marker process based on a bivariate Wiener model, Lifetime Data Analysis 1998, 4, 229-251.

[44] B. Xu, A. Oudalov, A. Ulbig, G. Andersson, and D.S. Kirschen, Modeling of Lithium-Ion Battery Degradation for Cell Life Assessment, IEEE Transactions on Smart Grid 2018, 9(2), 1131-1140.

[45] Z. Ye, M. Xie, L. Tang, and N. Chen, Semiparametric estimation of gamma processes for deteriorating products, Technometrics 2014, 56(4), 504-513.

[46] Z. Ye, M. Xie, L. Tang, and Y. Shen, Degradation-based burn-in planning under competing risks, Technometrics 2012, 54(2), 159-168.

[47] Z. Ye, and N. Chen, The inverse Gaussian process as a degradation model, Technometrics $2014,56(3), 302-311$ 